\title{
Artificial lung
}

\section{Francesco Petrella ${ }^{1,2}$, Lorenzo Spaggiari ${ }^{1,2}$}

${ }^{1}$ Department of Thoracic Surgery, European Institute of Oncology, Milan, Italy; ${ }^{2}$ Department of Oncology and Hemato-oncology, University of Milan, Milan, Italy

Contributions: (I) Conception and design: F Petrella; (II) Administrative support: F Petrella; (III) Provision of study materials or patients: F Petrella; (IV) Collection and assembly of data: F Petrella; (V) Data analysis and interpretation: F Petrella; (VI) Manuscript writing: All authors; (VII) Final approval of manuscript: All authors.

Correspondence to: Francesco Petrella, MD, PhD. Department of Thoracic Surgery, European Institute of Oncology, Via Ripamonti 435, Milan 20141, Italy. Email: francesco.petrella@ieo.it; francesco.petrella@unimi.it.

\begin{abstract}
Lung transplantation remains the definitive curative treatment for end-stage lung disease. However, future applications of tissue bioengineering could overcome the donor organ shortage and the need for immunosuppression. The final goal of lung tissue engineering is to recreate the whole spectrum of specialized lung tissues and thereby provide physiologic functions through bioengineered conducting airways, vasculature and gas exchange tissue. This review focuses on ongoing research in artificial lung development, open questions, achievements to date and how tissue engineering and stem cell technology may further contribute to the clinical application of bioartificial lungs. Although experimental transplantation of bioartificial lung developed by perfusing decellularized or synthetic scaffolds has been shown to provide gas exchange in vivo over a prolonged period, it should be clearly acknowledged that the development of a transplantable bioartificial lung is far from reality.
\end{abstract}

Keywords: Artificial lung; regenerative medicine; stem cell

Submitted Dec 12, 2017. Accepted for publication Dec 14, 2017.

doi: $10.21037 /$ jtd.2017.12.89

View this article at: http://dx.doi.org/10.21037/jtd.2017.12.89

\section{Introduction}

End-stage lung disease, namely chronic obstructive pulmonary diseases (COPD), represent the fourth leading cause of death worldwide (1). The increasing rates of tobacco smoking and exposure to air pollutants will further raise the number of COPD patients thus creating an urgent need for new therapeutic strategies.

Extracorporeal membrane oxygenation (ECMO) and mechanical ventilation can be temporarily used in this scenario as a bridge to lung transplantation that remains the only definitive treatment, but the need for immunosuppression and the donor organ shortage are major limits for a larger clinical impact (2).

Regenerative medicine, tissue engineering and stem cell technologies integrating the fields of engineering and biology may provide new treatment strategies for end organ failure, thereby obviating the problems of organ shortage, long-term immunosuppression and chronic rejection.

\section{Lung stem cell biology}

The lung has a considerable reparative capacity, when needed, in response to selective injuries and stimuli. The most likely hypotheses to explain this are that the lung could react to stress by activating stem cell populations or by reentering the cell cycle to repopulate damaged cells. Basal cells can function as tissue-specific stem cells of the airway epithelium in the proximal airway, while in the distal airway the bronchiolar epithelium is quiescent until damage occurs, when a subset of secretory cells, named variant club cells, show proliferation potential in response to injury. Type II alveolar epithelial cells are considered the ideal candidates for progenitor cells of the adult lung alveolus and some of them can proliferate, self-renew and form alveolar type I 
epithelial cells. At the transition from the bronchiolar to the alveolar region of the distal airway lies the bronchoalveolar duct junction where some variant club cells have airway epithelial regenerative potential after induced lung damage. These variant cells are known as bronchoalveolar stem cells although their existence in vivo has been contested (3).

\section{Lung mimicking devices}

Three-dimensional (3D) cell-cultures are the best models to reproduce the basic functions of lung physiology, overcoming many limits of 2D cell-culture systems that fail to reconstitute the in vivo cellular environment (4). Airliquid interface cultures are obtained by culturing patients' airway epithelial cells to model pathologic conditions like cystic fibrosis, asthma and tobacco-induced airway cell damage, thereby shedding more light on the underlying pathophysiology (5).

Organoids represent the evolution of $3 \mathrm{D}$ cell-culture models, relying on the properties of stem cells to differentiate into different specialized cell types and to self-organize into a $3 \mathrm{D}$ model with organ or tissuespecific properties and functions (6). Although pulmonary organoids deriving from one single stem cell have not yet been described, several studies have reported the creation of lung organoids from human pluripotent stem cells, primary respiratory cells and cell lines (7). The recent evolution of bioengineering led to the creation of "organson-a-chip", i.e., bioengineered devices reproducing tissue and organ properties and activities in a controlled environment (8). First described by Huh et al., the "lungon-a-chip" model reproduced the vascular-alveolar model by utilizing pulmonary epithelial cells exposed to air gases on one side and lung vascular endothelial cells exposed to flowing culture medium on the other side of a permeable synthetic membrane (9). These devices will probably be used in the near future as respiratory assist systems or oxygenators.

Tissue engineering is an interdisciplinary field applying the principles of engineering and biology to restore, repair or regenerate the functions of tissues and organs (10). It is based on the source and type of cells, type of scaffold, and composition of the extracellular matrix. In the complex field of airway and lung regeneration, several cases of tracheal bioengineering have been reported (11-14), but the attractive concept of bioengineered tracheal replacements has not yielded a definitive and reliable solution (15).

\section{Basic principles for artificial lung development}

The pivotal role in the process of artificial lung development is played by the synthetic interface through which gas exchange between blood and air takes place. This surface is made by the alveolar and vessel walls and has a total area of around $100 \mathrm{~m}^{2}$ in normal adult human lung (16). A sort of already existing artificial lung is the ECMO, a mechanical device routinely used since the 1950 s for respiratory support during cardiac surgery and critical care. Gas exchange is accomplished by the ECMO via a bundle of porous hollow fibers that nowadays can be extremely thin, thus effectively mimicking the human alveolar membrane (17). However, the polymers used in the fibers are thrombogenic, thus limiting ECMO activity to short-term clinical settings, but still with clot formation on the fiber surface (18).

Coating the ECMO membrane with human epithelial and endothelial cells could further mimic the native pulmonary vasculature. By antagonizing the ECMO's thrombogenic effects this could lead to miniaturized wearable ECMO devices, thus potentially resulting in a bioartificial lung (19-22).

\section{Lung tissue engineering}

The final goal of lung tissue engineering is to recreate the whole spectrum of specialized lung tissues and thereby provide physiologic functions through bioengineered conducting airways, vasculature and gas exchange tissue (2). One of the most challenging tasks in lung bioengineering is reproduction of the extracellular matrix, whose proteins are necessary for host-derived defense and graft homeostasis. Synthetic scaffolds provide gas exchange but lack extracellular matrix proteins and hence do not offer all the elements required for successful replacement of pulmonary function.

Another major problem to solve is the need to generate increasingly compact vascular flow networks capable of physiologic blood flow and gas exchange, thus recreating the lung's architectural hierarchy (2). One way to overcome these limits is human donor lung decellularization, providing exactly the complex hierarchical structure of vascular and airway lung architecture. Unfortunately, even this option presents several drawbacks: in case of incomplete recellularization, extracellular matrix proteins will be exposed and may initiate pathological reparative responses in vivo; disruption of the extracellular matrix 
during decellularization may result in scaffold degradation; lastly, as each decellularization process requires pre-existing native human lung, this approach does not solve the human donor shortage, thus making the use of xenogeneic scaffolds unavoidable (23).

\section{Lung scaffolds and cell type}

Decellularized pig lung represents the best model of xenogeneic scaffold as decellularized porcine tissue is already widely used in daily clinical practice for heart valves and vascular stents. However, to avoid the risk of zoonotic transmission and xenogeneic immunologic adverse reactions, pig lung needs extensive decellularization and rigorous testing of the donor beforehand (18).

Artificial scaffolds should be non-immunogenic, biocompatible, non-toxic, chemically stable and well tolerated by the host after implantation in order to avoid adverse reactions. Synthetic hydrogels such as polyethylene glycol (PEG) and polyvinyl alcohol (PVA) or synthetic elastomers such as poly glycerol sebacate (PGS) could be the ideal candidates to reproduce the complex lung architecture (24-26).

The most important cell families for artificial lung development are epithelial cells to line the airway lumen and endothelial cells to line the pulmonary vessels. To fully reproduce lung functions, several other types of cells are needed like alveolar macrophages, smooth muscle cells and others. As the lung has its own resident stem cells providing self-renewal in response to injury $(27,28)$, a possible approach to scaffold recellularization could be to stimulate the recipient's own repair mechanisms to home these cells with their correct spatial orientation within the decellularized or synthetic scaffold. Conversely, direct reseeding of the scaffold could be proposed by autologous stem cells, adult mesenchymal stromal cells being one of the best candidates for this purpose $(13,29,30)$.

\section{Conclusions}

Lung transplantation remains the definitive curative treatment for end-stage lung disease, but future applications of tissue bioengineering could overcome the donor organ shortage and the need for immunosuppression. Although experimental transplantation of bioartificial lung developed by perfusing decellularized or synthetic scaffolds has been shown to provide gas exchange in vivo over a prolonged period, it should be clearly acknowledged that its clinical application is still far from reality. As an alternative to artificial lungs, the lung stem cell pathway and plasticity may be targeted by novel compounds to stimulate their contribution to lung regeneration.

\section{Acknowledgements}

None.

\section{Footnote}

Conflicts of Interest: The authors have no conflicts of interest to declare.

\section{References}

1. Rabe KF, Hurd S, Anzueto A, et al. Global strategy for the diagnosis, management, and prevention of chronic obstructive pulmonary disease: GOLD executive summary. Am J Respir Crit Care Med 2007;176:532-55.

2. Song JJ, Ott HC. Bioartificial lung engineering. Am J Transplant 2012;12:283-8.

3. Petrella F, Rizzo S, Borri A, et al. Current Perspectives in Mesenchymal Stromal Cell Therapies for Airway Tissue Defects. Stem Cells Int 2015;2015:746392.

4. Schilders KA, Eenjes E, van Riet S, et al. Regeneration of the lung: Lung stem cells and the development of lung mimicking devices. Respir Res 2016;17:44.

5. Wark PA, Johnston SL, Bucchieri F, et al. Asthmatic bronchial epithelial cells have a deficient innate immune response to infection with rhinovirus. J Exp Med 2005;201:937-47.

6. Yin X, Mead BE, Safaee H, et al. Engineering Stem Cell Organoids. Cell Stem Cell 2016;18:25-38.

7. Nadkarni RR, Abed S, Draper JS. Organoids as a model system for studying human lung development and disease. Biochem Biophys Res Commun 2016;473:675-82.

8. Gjorevski N, Ranga A, Lutolf MP. Bioengineering approaches to guide stem cell-based organogenesis. Development 2014;141:1794-804.

9. Huh D, Matthews BD, Mammoto A, et al. Reconstituting Organ-Level Lung Functions on a Chip. Science 2010;328:1662-8.

10. Langer R, Vacanti JP. Tissue engineering. Science 1993;260:920-6.

11. Petrella F, Spaggiari L. Repair of large airway defects with bioprosthetic materials. J Thorac Dis 2017;9:3674-6.

12. Udelsman BV, Eaton J, Muniappan A, et al. Repair of 
large airway defects with bioprosthetic materials. J Thorac Cardiovasc Surg 2016;152:1388-97.

13. Aho JM, Dietz AB, Radel DJ, et al. Closure of a Recurrent Bronchopleural Fistula Using a Matrix Seeded With Patient-Derived Mesenchymal Stem Cells. Stem Cells Transl Med 2016;5:1375-9.

14. Macchiarini P, Jungebluth P, Go T, et al. Clinical transplantation of a tissue-engineered airway. Lancet 2008;372:2023-30.

15. Sjöqvist S, Jungebluth P, Lim ML, et al. Editorial Expression of Concern: Experimental orthotopic transplantation of a tissue-engineered oesophagus in rats. Nat Commun 2016;7:13310.

16. Galletti PM, Colton CK. Artificial lungs and blood-gas exchange devices. In: Bronzino JD. ed. The Biomedical Engineering Handbook. Boca Raton: CRC, 2000:1-19.

17. Bartlett RH. Extracorporeal life support: history and new directions. ASAIO J 2005;51:487-9.

18. Lemon G, Lim ML, Ajalloueian F, et al. The development of the bioartificial lung. Br Med Bull 2014;110:35-45.

19. Fritsche CS, Simsch O, Weinberg EJ, et al. Pulmonary tissue engineering using dual-compartment polymer scaffolds with integrated vascular tree. Int J Artif Organs 2009;32:701-10.

20. Polk AA, Maul TM, McKeel DT, et al. A Biohybrid artificial lung prototype with active mixing of endothelialized microporous hollow fibers. Biotechnol Bioeng 2010;106:490-500.

21. Wu ZJ, Zhang T, Bianchi G, et al. Thirty-day in-

Cite this article as: Petrella F, Spaggiari L. Artificial lung. J Thorac Dis 2018;10(Suppl 20):S2329-S2332. doi: 10.21037/ jtd.2017.12.89 vivo performance of a wearable artificial pump-lung for ambulatory respiratory support. Ann Thorac Surg 2012;93:274-81.

22. Garcia JP, Iacono A, Kon $\mathrm{ZN}$ et al. Ambulatory extracorporeal membrane oxygenation: a new approach for bridge-to-lung transplantation. J Thorac Cardiovasc Surg 2010;139:e137-9.

23. Badylak SF. Xenogeneic extracellular matrix as a scaffold for tissue reconstruction. Transpl Immunol 2004; $12: 367-77$.

24. Lutolf MP, Hubbell JA. Synthetic biomaterials as instructive extracellular microenvironments for morphogenesis in tissue engineering. Nat Biotechnol 2005;23:47-55.

25. Chen QZ, Liang SL, Thouas GA. Elastomeric biomaterials for tissue engineering. Prog Poly Sci 2013;38:584-671.

26. Wang Y, Ameer GA, Sheppard BJ, et al. A tough biodegradable elastomer. Nat Biotechnol 2002;20:602-6.

27. Rock JR, Hogan BL. Epithelial progenitor cells in lung development, maintenance, repair, and disease. Annu Rev Cell Dev Biol 2011;27:493-512.

28. Kajstura J, Rota M, Hall SR, et al. Evidence for human lung stem cells. N Engl J Med 2011;364:1795-806.

29. Petrella F, Spaggiari L, Acocella F, et al. Airway fistula closure after stem-cell infusion. $\mathrm{N}$ Engl J Med 2015;372:96-7.

30. Petrella F, Toffalorio F, Brizzola S, et al. Stem cell transplantation effectively occludes bronchopleural fistula in an animal model. Ann Thorac Surg 2014;97:480-3. 